\title{
Microvascular decompression in patients with isolated maxillary division trigeminal neuralgia, with particular attention to venous pathology
}

\author{
Raymond F. Sekula Jr., M.D., Andrew M. Frederickson, B.S., Peter J. Jannetta, M.D., \\ Sanjay Bhatia, M.D., Matthew R. Quigley, M.D., and Khaled M. Abdel Aziz, M.D. \\ Center for Cranial Nerve Disorders, Department of Neurosurgery, Allegheny General Hospital, Allegheny \\ Neuroscience Institute, and Drexel University College of Medicine, Pittsburgh, Pennsylvania
}

Object. The authors have the clinical impression that patients with isolated V2, or maxillary division, trigeminal neuralgia (TN) are most often women of a younger age with atypical pain features and a predominance of venous compression as the pathology. The aim of this study was to evaluate a specific subgroup of patients with V2 TN.

Methods. Among 120 patients who underwent microvascular decompression (MVD) for TN in 2007, data were available for 114; 6 patients were lost to follow-up. Patients were stratified according to typical (Burchiel Type 1), mixed (Burchiel Type 2a), or atypical (Burchiel Type 2b) TN. A pain-free status without medication was used to determine the efficacy of MVD. All patients were contacted in June 2008 and again in January 2009 at 12-24 months after surgery (median 18.4 months) and asked to rate their response to MVD as excellent (complete pain relief without medication), fair (complete pain relief with medication or some relief with or without medication), or poor (continued pain despite medication; that is, no change from their preoperative baseline pain status.

Results. Of 114 patients, 14 (12\%) had isolated V2 TN. Among these 14 were 2 typical (14\%), 1 mixed (7\%), and 11 atypical cases (79\%) of TN. Among the remaining 100 cases were 37 typical (37\%), 14 mixed (14\%), and 49 atypical cases (49\%) of TN. In the isolated V2 TN group, all patients were women as compared with $72 \%$ of women in the larger group of 100 patients ( $p=0.05$, chi-square test). The average age in the isolated V2 TN group was 51.2 years (median 48.1 years) versus 54.2 years (median 54.0 years) in the remainder of the group ( $\mathrm{p}=\mathrm{NS}$, unpaired Student t-test). In the isolated V2 TN group, there was a predominance of atypical pain cases (79\%) versus $49 \%$ in the remainder of the group, and this finding trended toward statistical significance ( $p=0.07$, chi-square test). Venous contact or compression (partly or wholly) was noted in $93 \%$ of the patients with isolated V2 versus $69 \%$ of the remainder of the group ( $\mathrm{p}=0.13$, chi-square test). The likelihood of excellent outcomes in the patients with V2 TN (71\%) was compared with that in typical pain cases (79\%) among patients in the rest of the group (that is, the bestoutcome group), and no difference was found between the 2 groups ( $\mathrm{p}=0.8$, chi-square test).

Conclusions. The authors confirmed that patients with isolated V2 TN were more likely to be female, tended toward an atypical pain classification with venous pathology at surgery, and fared just as well as those presenting with typical pain. (DOI: 10.3171/2009.8.FOCUS09156)

KEY WORDS • trigeminal neuralgia $\quad$ atypical pain $\quad$ vein $\quad$ isolated V2

$\mathrm{T}$ RIGEMINAL neuralgia is a debilitating facial pain disorder. Over the years, we have developed the clinical impression that patients with isolated V2, or maxillary division, pain are most often women of a younger age with atypical pain features and a predominance of venous compression as the pathology. Although the senior author has voiced this observation in previous reports, ${ }^{1,7}$ no dedicated study has been conducted to document the specific clinicopathological findings and out-

\footnotetext{
Abbreviations used in this paper: $\mathrm{MVD}=$ microvascular decompression; $\mathrm{SCA}=$ superior cerebellar artery; $\mathrm{SPV}=$ superior petrosal vein; $\mathrm{TN}=$ trigeminal neuralgia.
}

come of MVD in this group. Because MVD necessarily provides direct visualization of the root of the trigeminal nerve, the procedure affords an opportunity to better understand the clinicopathological correlates of vascular compression in this subgroup of patients with TN.

The safety and efficacy of MVD for TN has been established..$^{1,8,19,22}$ The aim of this study was to evaluate a specific subgroup of patients with isolated V2 TN while paying specific attention to clinical features, observed neurovascular pathology, and outcomes following MVD. By analyzing this specific subgroup, the pathophysiology and optimal treatment of isolated V2 TN may be better appreciated. 
TABLE 1: Summary of characteristics in 14 patients with isolated V2 TN*

\begin{tabular}{ccccccll}
\hline $\begin{array}{c}\text { Case } \\
\text { No. }\end{array}$ & $\begin{array}{c}\text { Age } \\
\text { (yrs) }\end{array}$ & Side & TN Classification & $\begin{array}{c}\text { Preop Duration of } \\
\text { Symptoms (yrs) }\end{array}$ & $\begin{array}{c}\text { Duration of } \\
\text { Follow-Up (mos) }\end{array}$ & Outcome & Offending Vessel \\
\hline 1 & 77 & It & atypical & 6 & 18 & excellent & SPV, SCA, arteriole \\
\hline 2 & 46 & rt & typical & 4 & 7 & excellent & SCA, vein \\
3 & 45 & rt & atypical & 2 & 13 & poor & SPV, SCA \\
4 & 64 & rt & atypical & 1 & 11 & fair & vein \\
5 & 62 & rt & atypical & 4 & 9 & excellent & SCA, vein \\
6 & 50 & It & atypical & $<1$ & 7 & excellent & SPV \\
7 & 32 & It & atypical & 1 & 10 & excellent & SPV, arteriole \\
8 & 58 & It & atypical & 3 & 14 & fair & SPV, SCA, AICA \\
9 & 48 & It & typical & $<1$ & 11 & excellent & vein \\
10 & 40 & rt & atypical & $<1$ & 7 & excellent & SCA \\
11 & 48 & It & atypical & 1 & 13 & excellent & SCA, SPV \\
12 & 43 & It & atypical & 2 & 12 & excellent & vein \\
13 & 78 & rt & mixed & 8 & 15 & poor & SCA, SPV \\
14 & 24 & It & atypical & $<1$ & 8 & excellent & vein \\
\hline
\end{tabular}

* All patients were women. Abbreviations: AICA = anterior inferior cerebellar artery; vein = unnamed vein.

\section{Methods}

This retrospective review of existing study materials was performed under a protocol approved by our institutional review board and in accordance with patient privacy rights protection as enforced by the Health Insurance Portability and Accountability Act.

Over a 12-month period from January 1, 2007, to December 31,2007, we prospectively collected information on all patients with TN who underwent evaluation at our center. The diagnosis of TN was made based on clinical history and physical examination. Magnetic resonance imaging of the brain with Gd confirmed the absence of structural lesions including tumors, arteriovenous malformations, and Chiari I malformations, and other confounding diagnoses (that is, multiple sclerosis) in each patient. Of the 120 patients who underwent MVD for TN in 2007, data were available for 114; 6 patients were lost to followup. Based on patient interviews, we stratified patients into 1 of 3 TN groups: typical (Burchiel Type 1 TN), mixed (Burchiel Type 2a TN), or atypical (Burchiel Type 2b $\mathrm{TN}){ }^{2,14}$ Typical TN was characterized by paroxysms of sharp and shooting episodic pain with a memorable onset and at least a partial response to anticonvulsants. At the other extreme, atypical TN pain was defined as beginning in an insidious manner and consisting predominately of a constant aching or burning pain that responded poorly to anticonvulsants. We defined all other cases as mixed TN, that is, having significant characteristics of both typical and atypical TN.

Following MVD, collected data included information derived from office visits, operative drawings and videos, and telephone follow-ups. In this study, a pain-free status without medication was used to determine the efficacy of MVD. All patients were contacted in June 2008 and again in January 2009 at 12-24 months after surgery (median 18.4 months). Outcome was determined by a disinterested observer (A.M.F.), and during telephone inter- views, patients were asked to characterize their outcomes as "complete pain relief without medication" (excellent), "complete pain relief with medication" (fair), "some relief with or without medication" (fair), or "continued baseline pain despite medication" (poor).

\section{Operative Procedure}

Microvascular decompression was performed with the patient under general anesthesia in the contralateral decubitus position and utilizing auditory brainstem evoked potentials in a manner previously described..$^{13} \mathrm{~A}$ retromastoid incision was made behind the hairline, and a craniectomy about the asterion was performed. The intersection of the transverse sinus and sigmoid sinuses was identified, and the dura mater was opened. After appropriate brain relaxation was achieved with CSF drainage, the cerebellum was gently elevated, and the trigeminal nerve was identified and examined for vascular contact at the root entry zone. Arterial vessels and veins similar or larger in diameter to the SCA were decompressed using shredded Teflon pledgets. Veins that were smaller in diameter than the SCA were carefully separated from the trigeminal nerve, cauterized using bipolar cautery, and divided with microscissors.

\section{Statistical Analysis}

Statistical analysis was performed with MEDCALC 9.001 for PC.

\section{Results}

\section{Patient Characteristics}

Of 114 patients who underwent MVD, 14 (12\%) had isolated V2 TN. Among these 14 were 2 typical (14\%), 1 mixed (7\%), and 11 atypical cases (79\%) of TN (Table 1). Among the remaining 100 cases were 37 typical (37\%), 


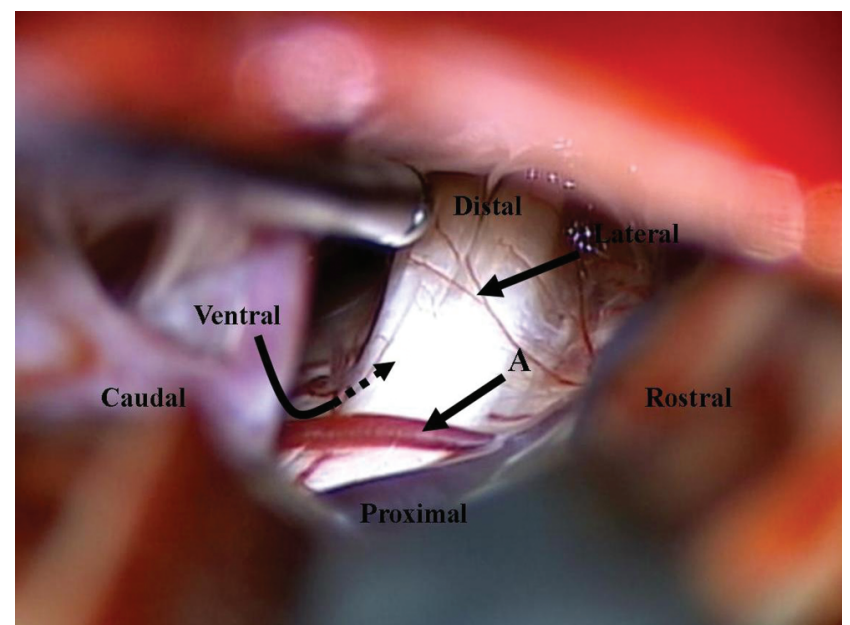

FIG. 1. Case 9. Photograph depicting the ventral aspect of the left trigeminal nerve, which faces the pons, and the lateral aspect, which faces away from the pons. Note the example of a transverse pontine vein (a tributary of the SPV, A) compressing the lateral aspect of the right trigeminal nerve. This patient had no other vascular compression, and after decompression of the vein at the last follow-up she remained pain free without medication.

14 mixed (14\%), and 49 atypical cases (49\%) of TN. These distributions were not significantly different $\left(\chi^{2}=0.11\right)$. All patients in the isolated V2 TN group were women as compared with $72 \%$ women among those who were not ( $p=0.05$, chi-square test). The average age of the isolated V2 TN group was 51.2 years (median 48.1 years) versus 54.2 years (median 54.0 years) in the larger group ( $p=$ NS, unpaired Student t-test). In the isolated V2 TN group, there was a predominance of atypical pain cases (79\%) versus $49 \%$ in the remainder of the group, and this result trended toward statistical significance $(\mathrm{p}=0.07$, chisquare test).

\section{Operative Findings and Outcomes}

Venous compression (partly or wholly) was noted in 93\% of the isolated V2 TN group versus $69 \%$ in the remaining 100 cases ( $p=0.13$, chi-square test; Fig. 1$)$. At the time of follow-up (median 18.4 months, range 12-24 months), 10 patients (71\%) reported an excellent outcome, $2(14 \%)$ a fair outcome, and $2(14 \%)$ a poor outcome. Of the 10 patients reporting an excellent outcome, 1 required a second MVD (a few days after the first MVD) for a "missed" anterior inferior cerebellar artery compressing the nerve caudally as well as the SCA and SPV that were decompressed at the first MVD. Overall, venous pathology was noted in 13 of 14 cases, with involvement of the SPV tributaries (most commonly the transverse pontine vein) in 7 patients, an aberrant trigeminal vein in 1, and unnamed veins in 5 . Five of 14 patients with isolated V2 $\mathrm{TN}$ had isolated venous compression of the trigeminal nerve, and 4 of these 5 reported an excellent outcome following treatment, whereas 1 patient reported a fair outcome. Nine patients had rostral compression and 4 had caudal compression. In addition, the veins were ventral to the nerve in 5 patients and dorsal to the nerve in 6 patients. Two veins coursed between the motor and sensory root of the trigeminal nerve. In 6 patients, the venous pathology was present at the proximal portion of the nerve in close association with the root entry zone of the nerve.

We compared the likelihood of excellent outcomes in all patients with V2 TN (71\%) versus that in patients with typical pain (79\%) from among the remaining 100 cases (that is, the best-outcome group) and found that there was no difference between the 2 groups $(p=0.8$, chi-square test). Among the patients who had typical pain, 2 (100\%) of 2 had excellent outcomes. Eight (73\%) of 11 patients with atypical TN experienced excellent outcomes, whereas $2(18 \%)$ of 11 and $1(9 \%)$ of 11 with atypical TN had fair and poor outcomes, respectively. One patient with mixed TN reported a poor outcome following MVD. A percutaneous glycerol rhizotomy was later performed, but the procedure failed to relieve her pain.

\section{Discussion}

Without modern magnification, Dandy ${ }^{3}$ noted venous compression of the trigeminal nerve in $14 \%$ of his cases of TN. Since Dandy's report, authors of several studies have noted an increased rate of venous contact or compression in patients with $\mathrm{TN}$ as compared with rates in cadavers without a history of TN.,5 Trigeminal neuralgia, which is most often unilateral, can occur in any one or combination of the 3 branches of the trigeminal nerve. In order of frequency in the general population, TN occurs in the V2 and V3 distributions (30-36\%), V2 distribution (10-22\%), V1 and V2 distributions (17-22\%), V3 distribution (15\%), V1 and V2 and V3 distributions (8-12\%), and V1 distribution (2-3\%)., 1,19 Trigeminal neuralgia occurs more commonly in women for reasons that are poorly understood, ${ }^{1}$ and in the cohort of patients with isolated V2 TN in the present study, all were women, which was statistically significant. Contrary to our hypothesis, however, no difference in age was found between the isolated V2 TN cohort and the remaining 2007 TN cohort. This finding may reflect the preponderance of atypical cases (more often younger women) in our 2007 TN cohort.

In our series, a higher proportion of patients with isolated V2 TN met the criteria for atypical TN (11 patients [79\%]) than did those in the rest of the $2007 \mathrm{TN}$ cohort (49 patients [49\%]). Despite well-intentioned attempts at classifying the various forms of facial pain, no classification scheme has been uniformly accepted. The majority of practitioners continue to divide TN into typical (that is, tic douloureux) and atypical forms or use the Burchiel classification scheme. ${ }^{2,20}$ Recently, some authors have proposed the term "transitional TN." ${ }^{3}$ While many cases of TN do transition from strictly typical to atypical $\mathrm{TN},{ }^{2}$ others begin with constant background pain (usually "burning") with crescendos or paroxysms of lancinating pain throughout the day and do not fit this transitional subgroup. Most recently, in response to Heros, ${ }^{6}$ Burchiel wrote, "Type 2 TN may well be a composite category, containing one or more other diagnoses. The results of our study would tend to support this thesis. Perhaps, it would make more sense to divide Type 2 TN into 2 subdivisions: cases in which there is some history of lancinating pain (Type $2 \mathrm{a}$ ), and those in which there is no such 
history (Type 2b)."14 For similar reasons, our group has referred to all instances of TN characterized by considerable components of both typical and atypical TN as "mixed." In the future, despite the additional complexity, we may need to further classify multiple subgroups within Type 2 TN.

The initial treatment of all forms of TN is most often medical. ${ }^{24}$ When adequate relief of pain cannot be achieved with medication, other treatments should be considered. Confusion and disagreement persist, however, regarding the most appropriate treatment for medically refractory TN. Because of its high rate of success, MVD is considered by many to be the optimal initial treatment for typical TN in young and elderly otherwise healthy adults.,17 In considering MVD for TN, the utility of distinguishing between the various subtypes of $\mathrm{TN}$ is becoming clearer. In reviewing Jannetta's ${ }^{7}$ results over a 24-year period, Tyler-Kabara et al. ${ }^{23}$ have shown that patients with typical TN fare far better than those with atypical TN. Li et al. ${ }^{10}$ have suggested that patients with typical TN of a shorter duration fare better following MVD. Note, however, that Sindou et al. ${ }^{19}$ have reported equivalent rates of success with MVD in a consecutive series of patients with typical and atypical TN. A close examination of the inclusion and exclusion criteria in their study may help to clarify these results. While 2232 patients were evaluated for surgery for TN, only 362 were "randomized" to MVD and 1680 were "randomized" to thermal rhizolysis. In fact, only those patients with typical or atypical TN characterized "as paroxysmal pain (at least at the beginning of the disease) but later accompanied by a constant deep aching or burning pain" were offered MVD. Moreover, all patients, at least initially, responded to anticonvulsants. In effect, Sindou et al. have reported outcomes in a highly selected series of patients with typical and so-called transitional TN. With this selection in mind, one cannot conclude from their report that typical and atypical TN (as classified by most clinicians) respond equally well to MVD. Based on their report, however, one can conclude that typical and transitional TN respond equally well to MVD. Similarly, Sandell et al. ${ }^{16}$ have recently shown that patients with variable degrees of constant pain fared as well as those without constant pain. Like Sindou et al., however, Sandell and colleagues only included patients with constant pain and whose TN started with purely episodic pain (that is, Type 1, or typical, and transitional TN). Most recently, Miller et al. ${ }^{14}$ have provided more evidence that patients with Type 1, or typical, TN fare better with MVD and acknowledged that further partitioning of Type 2 , or atypical, TN may be useful. In most cases, outcomes of MVD for TN appear to be strongly related to exclusion, classification, and outcome criteria. Results from the present series of patients with typical TN compare favorably with data from our prior studies with similar exclusion, classification, and outcome criteria. ${ }^{1}$

In light of the above findings, many patients with atypical TN are denied MVD because of the inferior results as compared with the outcomes in those with typical $\mathrm{TN}$. Therefore, some practitioners offer ablative procedures (that is, balloon rhizolysis, glycerol rhizotomy, thermal rhizolysis, or radiosurgical rhizolysis) first to those suffering from atypical $\mathrm{TN}$, with stereotactic radiosurgical rhizolysis increasingly offered as a first-line treatment despite marginal results. ${ }^{11,15,18}$ Although patients with typical, or Type 1, TN fare better than those with atypical, or Type 2, TN generally with any treatment, subgroups with atypical TN that are more responsive to MVD do exist, such as the group of patients with isolated V2 TN in the present study. In addition, there is no evidence to suggest that ablative treatments provide superior pain relief from atypical TN over MVD. Most importantly, the risk of adverse consequences relative to the trigeminal nerve is far higher with ablative procedures. ${ }^{21}$

Notably, only 1 patient in the isolated V2 TN group had a poor outcome following MVD. She stated that the surgery relieved the atypical, constantly dull pain in the V2 distribution, but it did not relieve the excruciating stabbing pain that was the source of most of her troubles. It is notable that this patient was the only 1 of 14 who had undergone stereotactic radiosurgical rhizolysis (that is, Gamma Knife) and instead received an 80-Gy dose to the nerve 12 months prior to MVD. Importantly, despite a predominance of patients with atypical TN (79\%) in our isolated V2 TN group, the outcomes of patients with atypical, isolated V2 TN were as good as those with typical TN of all distributions. The small number of patients in our study remains a limitation, and in the future we intend to add to this series and reassess our results in patients with isolated V2 TN.

Veins are frequently compressive in TN (20-68\% of cases). ${ }^{1,12,19}$ In our isolated V2 TN cohort, the incidence of venous contact or compression (with or without additional arterial compression) was higher at 93\%. Although arterial compression remains the dominant pathology in $\mathrm{TN}$, the role of veins in this disorder is being increasingly recognized..$^{9,12}$ In our series of patients with isolated V2 TN, 4 had compression by a sole vein, and 3 of 4 patients continue to report an excellent outcome following treatment of a sole vein. The knowledge that patients with isolated V2 TN can have higher rates of venous compression could aide surgeons in their exploration and treatment of the trigeminal nerve in this particular subset of patients. Nonetheless, the difference in venous compression between the patients with isolated V2 TN and all others from 2007 with TN did not meet the level of statistical significance as posited in our hypothesis. This result may reflect a true lack of significance, insufficient statistical power, or an inability to determine such a difference because we uniformly decompress the trigeminal nerve of all compression (arterial or venous) no matter how seemingly insignificant a vessel may appear. In the 2007 cohort, veins were decompressed in $69 \%$ of the patients (with or without additional arterial compression). This practice reflects our belief that "missed" veins at the time of initial surgery account for a large proportion of MVD failures and that the most common cause of TN recurrence is due to recollateralization or development and the growth of veins. ${ }^{9}$ To date, however, the role of veins specifically in atypical, or Type $2 \mathrm{~b}, \mathrm{TN}$ has received limited attention. Matsushima et al. ${ }^{12}$ have noted 7 cases of venous compression among a series of 121 patients with typical TN. Although the results of venous decompres- 


\section{Microvascular decompression for isolated V2 trigeminal neuralgia}

sion in these 7 cases were excellent, patients with atypical, or Type $2 \mathrm{~b}, \mathrm{TN}$ were specifically excluded. Nonetheless, it is reasonable to assume that veins can cause both typical and atypical TN. Furthermore, our understanding of the surgical management of venous compression in TN is evolving. At this time, we cauterize those veins with a smaller diameter than the SCA, and we decompress with Teflon pledgets those veins with a larger diameter than the SCA.

\section{Conclusions}

An understanding of the likely neurovascular pathology responsible for each particular cranial rhizopathy and the outcomes associated with MVD will aid in the recognition and formulation of an appropriate treatment plan for each patient.

In this study, we confirmed that patients with isolated V2 TN were more likely to be female, tended toward an atypical pain classification with venous pathology, and fared just as well as those presenting with typical pain. Because our results in this small subset of patients are notable, especially in patients with atypical, or Type $2, \mathrm{TN}$, it is likely that meticulous attention to venous pathology may result in better outcomes for all patients with TN.

\section{Disclaimer}

The authors of this manuscript have no financial interest in the subject or technical devices under discussion.

\section{References}

1. Barker FG Jr, Jannetta PJ, Bissonette DJ, Larkins MV, Jho HD: The long-term outcome of microvascular decompression for trigeminal neuralgia. N Engl J Med 334:1077-1083, 1996

2. Burchiel KJ: A New Classification for Facial Pain. Neurosurgery 53:1164-1167, 2003

3. Dandy W: Concerning the cause of traigeminal neuralgia. Am J Surg 24:447-455, 1934

4. Haines SJ, Jannetta PJ, Zorub DS: Microvascular relations of the trigeminal nerve. An anatomical study with clinical correlation. J Neurosurg 52:381-386, 1980

5. Hamlyn PJ: Neurovascular relationships in the posterior cranial fossa, with special reference to trigeminal neuralgia. 2 . Neurovascular compression of the trigeminal nerve in cadaveric controls and patients with trigeminal neuralgia: quantification and influence of method. Clin Anat 10:380-388, 1997

6. Heros RC: Results of microvascular decompression for trigeminal neuralgia. J Neurosurg 110:617-619, 2009

7. Jannetta PJ: Surgical treatment of cranial rhizopathies, in Grady MS (ed): Congress of Neurological Surgeons. Montreal, Quebec, Canada: Williams \& Wilkins, 1996, Vol 44, pp 181-195

8. Kondo A: Follow-up results of microvascular decompression in trigeminal neuralgia and hemifacial spasm. Neurosurgery 40:46-42, 1997

9. Lee SH, Levy EI, Scarrow AM, Kassam A, Jannetta PJ: Recurrent Trigeminal Neuralgia Attributable to Vein after Microvascular Decompression. Neurosurgery 46:356-362, 2000
10. Li ST, Pan Q, Liu N, Shen F, Liu Z, Guan Y: Trigeminal neuralgia: what are the important factors for good operative outcomes with microvascular decompression. Surg Neurol 62:400-405, 2004

11. Maesawa S, Salame C, Flickinger JC, Pirris S, Kondziolka D, Lunsford LD: Clinical outcomes after stereotactic radiosurgery for idiopathic trigeminal neuralgia. J Neurosurg 94: 14-20, 2001

12. Matsushima T, Huynh-Le P, Miyazono M: Trigeminal neuralgia caused by venous compression. Neurosurgery 55:334339,2004

13. McLaughlin MR, Jannetta PJ, Clyde BL, Subach BR, Comey $\mathrm{CH}$, Resnick DK: Microvascular decompression of cranial nerves: lessons learned after 4400 operations. J Neurosurg 90:1-8, 1999

14. Miller JP, Magill ST, Acar F, Burchiel KJ: Predictors of longterm success after microvascular decompression for trigeminal neuralgia. J Neurosurg 110:620-626, 2009

15. Regis J, Metellus P, Hayashi M, Roussel P, Donnet A, BilleTurc F: Prospective controlled trial of gamma knife surgery for essential trigeminal neuralgia. J Neurosurg 104:913-924, 2006

16. Sandell T, Eide PK: Effect of microvascular decompression in trigeminal neuralgia patients with or without constant pain. Neurosurgery 63:93-100, 2008

17. Sekula RF, Marchan EM, Fletcher LH, Casey KF, Jannetta PJ: Microvascular decompression for trigeminal neuralgia in elderly patients. J Neurosurg 108:689-691, 2008

18. Sheehan J, Pan HC, Stroila M, Steiner L: Gamma knife surgery for trigeminal neuralgia: outcomes and prognostic factors. J Neurosurg 102:434-441, 2005

19. Sindou M, Leston J, Howeidy T, Decullier E, Chapuis F: Micro-vascular decompression for primary Trigeminal Neuralgia (typical or atypical). Long-term effectiveness on pain; prospective study with survival analysis in a consecutive series of 362 patients. Acta Neurochir (Wien) 148:1235-1245, 2006

20. Sweet W: Atypical facial neuralgia, in White J, Sweet W (eds): Pain and the Neurosurgeon. Springfield, IL: Charles C Thomas, 1969

21. Taha J, Tew J: Comparison of surgical treatments for trigeminal neuralgia: reevaluation of radiofrequency rhizotomy. Neurosurgery 38:865-871, 1996

22. Theodosopoulos PV, Marco E, Applebury C, Lamborn KR, Wilson CB: Predictive model for pain recurrence after posterior fossa surgery for trigeminal neuralgia. Arch Neurol 59:1297-1302, 2002

23. Tyler-Kabara EC, Kassam AB, Horowitz MH, Urgo L, Hadjipanayis C, Levy EI, et al: Predictors of outcome in surgically managed patients with typical and atypical trigeminal neuralgia: comparison of results following microvascular decompression. J Neurosurg 96:527-531, 2002

24. Zakrzewska JM, Patsalos PN: Long-term cohort study comparing medical (oxcarbazepine) and surgical management of intractable trigeminal neuralgia. Pain 95:259-266, 2002

\section{Manuscript submitted July 12, 2009.}

Accepted August 27, 2009.

Address correspondence to: Raymond F. Sekula Jr., M.D., Center for Cranial Nerve Disorders, Department of Neurosurgery, Allegheny General Hospital, 420 East North Avenue, Suite 302, Pittsburgh, Pennsylvania 15212. email: rsekula@wpahs.org. 